\title{
Comparación de los niveles de ansiedad y depresión entre estudiantes del último año de medicina de la UAA e internos de pregrado del estado de Ags.
}

Terrones-Saldívar MC*, Rosas-Cabral A*, García-Huízar P*, Moreno-Castanedo H*, Prieto-Macías J*, Reyes-Robles ME*.

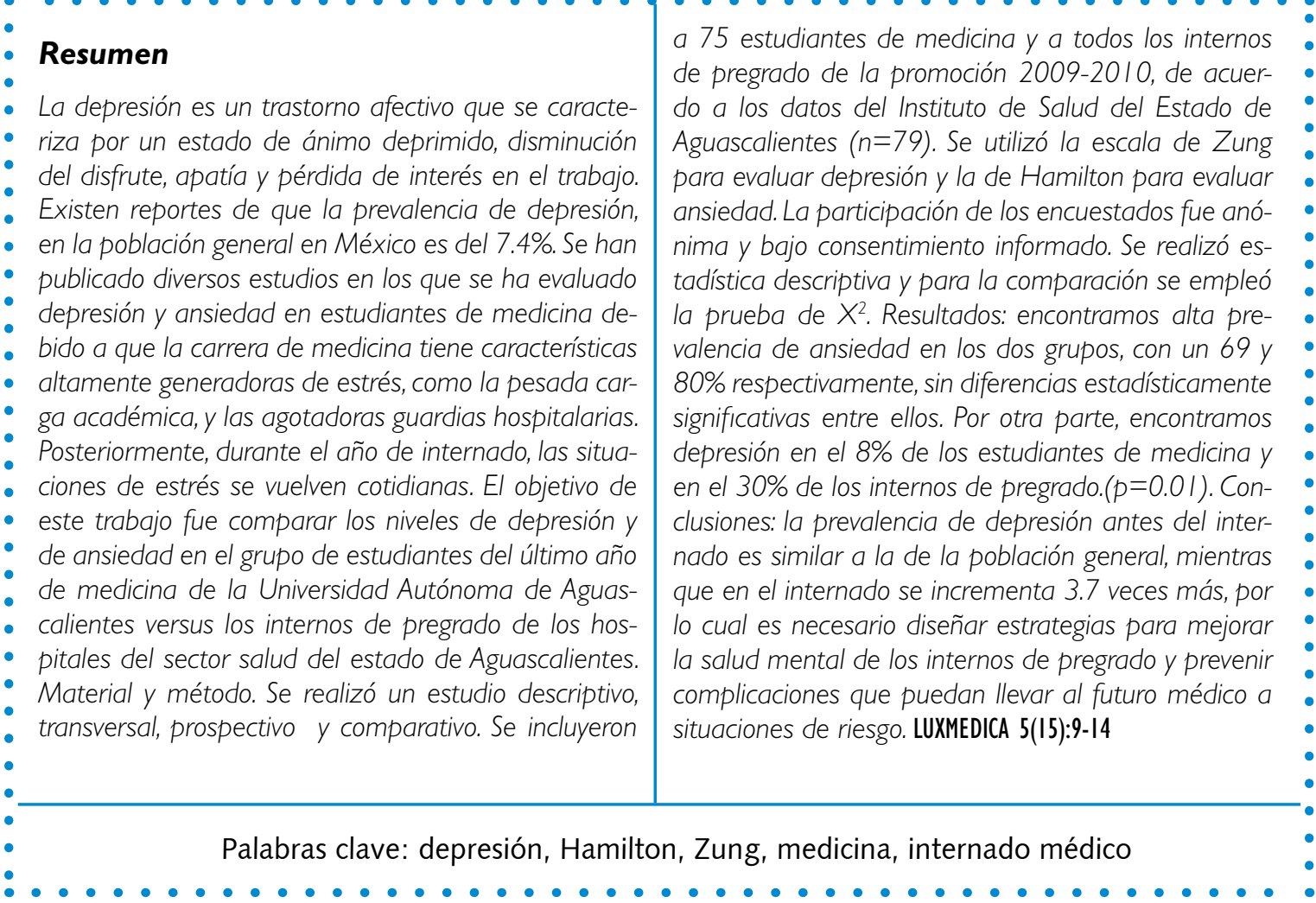

* Profesores investigadores de medicina del Centro de Ciencias de la Salud, Universidad Autónoma de Aguascalientes.

Fecha de recibido: 31-junio-2010

Fecha de aceptado: 12-agosto-2010

Correspondencia: Terrones Saldivar Ma. del Carmen. Doctora en ciencias, Profesor investigador Depto de Gineco-Obstetricia y Pediatría, Centro de Ciencias de la Salud, UAA. Av. Universidad \#910 Ciudad Universitaria Ags., Ags. CP 20100 Tel. 9108443 dra_terr@yahoo.com 


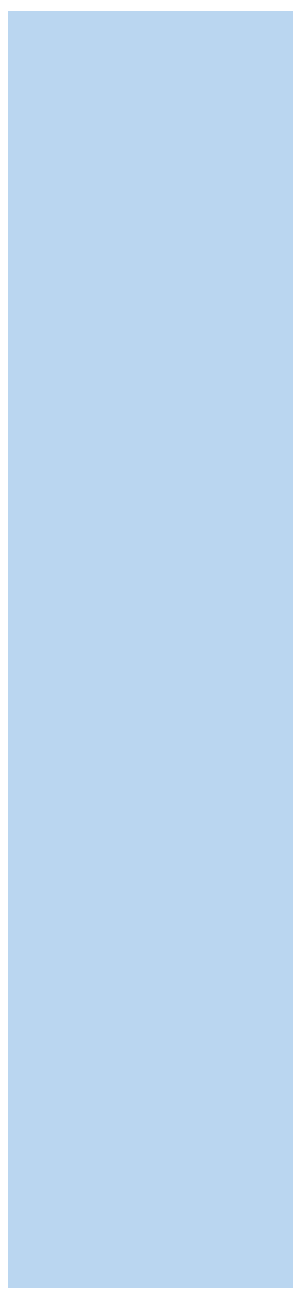

\section{Antecedentes}

La depresión es un trastorno afectivo que se caracteriza por un estado de ánimo deprimido, disminución del disfrute, apatía y pérdida de interés en el trabajo, distorsión de los hábitos fundamentales de todo ser humano, generándose insomnio, anorexia, disminución de la libido, de la capacidad de interacción con sus semejantes, irritabilidad, ansiedad y frecuentemente ideación suicida. ${ }^{1}$ Es una entidad clínica que ocupa uno de los primeros lugares como motivo de consulta en los servicios de psiquiatría. ${ }^{2}$ La Organización Mundial de la Salud (OMS) ha informado que la depresión ocupa la segunda causa de discapacidad a nivel mundial y afecta aproximadamente a 121 millones de personas. ${ }^{3}$ Se calcula que la prevalencia de depresión en México es del $7.4 \%$. Existen diversos resultados sobre la salud mental en estudiantes universitarios, especialmente en los estudiantes de medicina, donde se ha evaluado la ansiedad y la depresión..$^{4-7}$ Algunos autores establecen que antes de entrar a la escuela de medicina, el estatus emocional de los estudiantes se parece al de la población general. La carrera de medicina tiene características altamente generadoras de estrés, como la pesada carga académica, y las agotadoras guardias hospitalarias. Posteriormente, durante el año de internado, las situaciones de estrés se vuelven cotidianas. Poco se ha trabajado para identificar los problemas emocionales que tienen durante su entrenamiento los estudiantes de medicina y los internos de pregrado. El objetivo de este trabajo fue comparar los niveles de depresión y de ansiedad en el grupo de estudiantes del último año de medicina de la Universidad Autónoma de Aguascalientes versus los internos de pregrado de los hospitales del sector salud del estado de Aguascalientes.

\section{| | | | | | | | | | | | | | | | | | | | | | | | | | | | | | | | | | | | | | | | | | | | | | | | | | | | | | | | | | | | | | | | | | | | | | | | | | | | | | | | | | | | | | | | | | | | | | | | | | | | | | | | | | | | | | | ||}

\section{Metodología}

Se realizó un estudio descriptivo, transversal, prospectivo y comparativo. Se incluyeron en el grupo 1 a todos los estudiantes del noveno y décimo semestre de la carrera de medicina de la UAA $(n=75)$ y en el grupo 2, a todos los internos de pregrado de la promoción 2009-2010 de acuerdo a los datos del Instituto de Salud del Estado de Aguascalientes $(n=79)$. Se utilizó la escala de Zung para evaluar la depresión y la de Hamilton para evaluar la ansiedad. La participación de los encuestados fue anónima y bajo consentimiento informado. A los estudiantes de medicina, el instrumento fue autoaplicado en el salón de clase y para los internos de pregrado en cada una de las sedes hospitalarias correspondientes, con el apoyo de los jefes de enseñanza de cada hospital. El instrumento autoaplicado de depresión de Zung evalúa algunos de los síntomas de la depresión. El cuestionario consta de 20 afirmaciones, a las que se les da un puntaje que va de 1 a 4 dependiendo de la respuesta: 1 para la respuesta nunca o muy pocas veces; 2 para algunas veces, 3 para frecuentemente y 4 para siempre. En las preguntas 2,5,6,11,1 $2,14,16,17,18$,y 20 se invierte el orden del puntaje. El puntaje para evaluar la severidad de los síntomas fueron los siguientes: hasta 49 puntos, sin síntomas; más de 50 
puntos, depresión leve ó mínima; más de 60 puntos, depresión moderada y más de 70 puntos, depresión severa. La escala de autoevaluación de ansiedad de Hamilton, consta de 14 ítems, a los que se les da un puntaje de 0 a 4 dependiendo de la respuesta: 0 para ausente; 1 para leve; 2 para moderado; 3 para grave y 4 para muy gra- ve. La interpretación del puntaje fue: menos de 5 puntos, sin síntomas; de 6 a 14 puntos, ansiedad leve y más de 15 puntos, ansiedad moderada a grave. Los resultados se expresan en porcentajes y para determinar si hay diferencias en la proporción de manifestaciones depresivas entre los dos grupos, se utilizó la prueba de $x^{2}$.

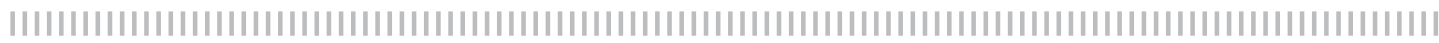

\section{Resultados}

En el grupo 1, conformado por 43 estudiantes del noveno semestre de medicina y por 32 estudiantes del décimo semestre (total de 75) encontramos una alta prevalencia de ansiedad, con un 69\% (52/75) del total de estudiantes. De los 52 estudiantes identificados con ansiedad, 30 de ellos (57\%) calificaron para ansiedad leve y el resto, 22 estudiantes, (43\%) para ansiedad moderada-severa. Para el grupo 2, integrado por los internos de pregrado de seis sedes hospitalarias diferentes, encontramos de igual forma, una mayor prevalencia de ansiedad, con un 80\% (58/72). De éstos, 35 (60\%) calificaron para ansiedad leve y el resto, 23 (40\%) para ansiedad moderada-severa. Al comparar ambas prevalencias, no encontramos diferencias significativas. Solo una tendencia al incremento de la ansiedad en el grupo de internos de pregrado. Es de llamar la atención, que en todas las unidades sede de internado de pregrado, encontramos ansiedad, con un rango que varió entre el 50 y el $90 \%$. (ver tabla 1). En cuanto a depresión, encontramos diferencias significativas cuando comparamos la prevalencia de depresión entre el grupo de estudiantes de medicina, $8 \%(6 / 75)$ versus la prevalencia de depresión entre los internos de pregrado, 24\% $(24 / 79)(p<0.01)$ (gráfica 1). En ningún caso encontramos depresión severa, ni entre los estudiantes del último año de medicina ni tampoco entre los internos de pregrado. También nos llamó la atención que en uno de los hospitales, ningún encuestado resultó con problemas de depresión. Para el resto de las instituciones sede de internado, la prevalencia de depresión varió entre el 6 y el $66 \%$. En dos de los seis hospitales, donde encontramos alta prevalencia de ansiedad, fueron en los mismos que se encontró la mayor prevalencia de depresión. (tabla 2)

\section{Tabla I}

Frecuencias de ansiedad entre los estudiantes de medicina de la UAA y los internos de pregrado del estado de Aguascalientes.

\begin{tabular}{|c|c|c|c|c|}
\hline & \multicolumn{2}{|c|}{$\begin{array}{l}\text { Estudiantes medicina } \\
\qquad(n=75)\end{array}$} & \multicolumn{2}{|c|}{$\begin{array}{l}\text { Internos pregrado } \\
\qquad(n=72)\end{array}$} \\
\hline Sin ansiedad & $23 / 75(31 \%)$ & & $14 / 72(20 \%)$ & \\
\hline Con ansiedad & $52 / 75(69 \%)$ & $\begin{array}{l}\text { Leve }(30 / 52)(57 \%) \\
\text { Moderada-severa } \\
22 / 52(43 \%)\end{array}$ & $58 / 72(80 \%)$ & $\begin{array}{l}\text { Leve }(35 / 58)(60 \%) \\
\text { Moderada-severa } \\
(23 / 58)(40 \%)\end{array}$ \\
\hline
\end{tabular}




\section{Tabla 2}

\section{Frecuencias de depresión entre los estudiantes de medicina de la UAA y los internos de pregrado del es-} tado de Aguascalientes.

\begin{tabular}{|c|c|c|c|c|}
\hline & \multicolumn{2}{|c|}{$\begin{array}{l}\text { Estudiantes medicina } \\
\qquad(n=75)\end{array}$} & \multicolumn{2}{|c|}{$\begin{array}{c}\text { Internos pregrado } \\
(n=79)\end{array}$} \\
\hline Sin depresión & $69 / 75(92 \%$ & & $5 / 79(70 \%)$ & \\
\hline Con depresión & $6 / 75(8 \%)$ & $\begin{array}{l}\text { Leve } 4 / 6 \\
\text { Moderada } 2 / 6 \\
\text { Severa } 0\end{array}$ & $24 / 79(30 \%)^{* *}$ & $\begin{array}{l}\text { Leve } 24 / 24 \\
\text { Moderada } 0 \\
\text { Severa } 0\end{array}$ \\
\hline
\end{tabular}

$$
{ }^{* *} p<0.001
$$

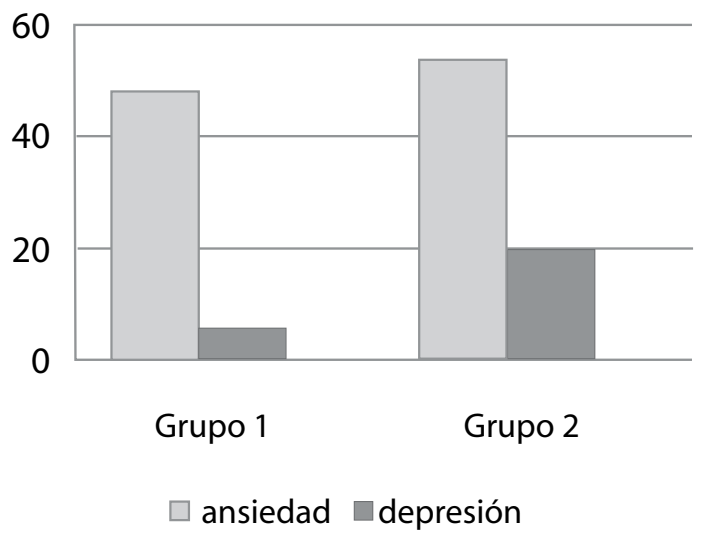

Gráfica 1. Distribución de la prevalencia de ansiedad y depresión en el grupo de estudiantes de medicina del último año de la carrera de medicina de la UAA (grupo 1) y en el grupo de internos de pregrado del estado de Aguascalientes (grupo 2).

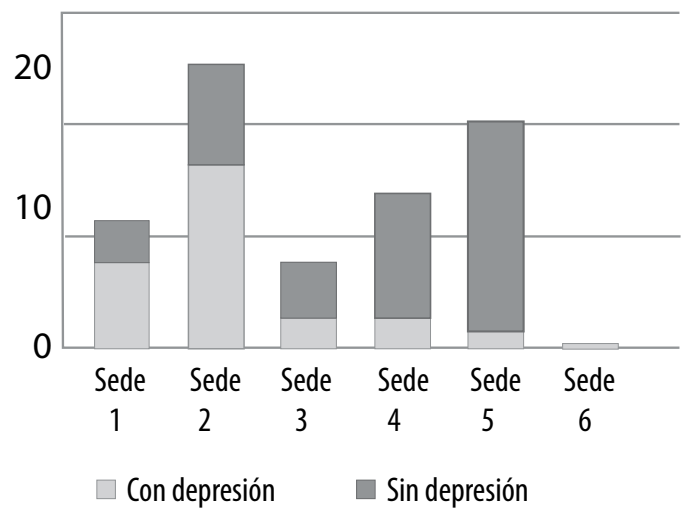

Gráfica 2. Distribución de internos con y sin depresión por sede hospitalaria.

\section{||||||||||||||||||||||||||||||||||||||||||||||||||||||||||||||||||||||||||||||||||||||||||||||||||||||||||||||||||||||}

\section{Discusión}

La depresión y la ansiedad son trastornos mentales con elevada frecuencia en la población general de nuestro país y tienen una clara tendencia hacia incrementar su incidencia ${ }^{1,2}$. Las condiciones generales de la vida actual constituyen factores relevantes para su incremento y limitan de manera importante el bienestar general de los pacientes. $^{8}$

A pesar de ser un padecimiento con elevada prevalencia en la población general, es un hecho bien reconocido, que los estudiantes de medicina tienen agregado un importante componente de estrés, debido, entre otros factores, a la elevada carga académica, la presión constante para alcanzar el éxito y a la fuerte competencia con sus compañeros. ${ }^{9-12}$ Esto se hace evidente, ya que algunos autores han demostrado que el nivel de ansiedad que tienen los estudiantes, durante el primer año de su estancia en la carrera de medicina es significativamente mayor que el que tienen antes de ingresar a la carrera ${ }^{8,9}$, además los estudiantes de medicina tienen mayores 
niveles de estrés que sus pares por edad estudiando otras licenciaturas ${ }^{13}$.

También otros autores han reportado que los niveles de estrés son mayores en los estudiantes de medicina comparado con los de otras carreras debido a que la carga de trabajo es más elevada que en otras licenciaturas ${ }^{14}$. Se ha propuesto, que los primeros años de la carrera son los que mayor estrés generan en los alumnos, debido principalmente al importante cambio en el estilo de vida que representa el paso de la educación media superior a la carrera de medicina ${ }^{15}$.

Otros autores han sugerido que el mayor estrés se da en la transición de los ciclos básicos a los ciclos clínicos, debido a que sufren una pérdida del control externo, dado por la seguridad de sus conocimientos en el área básica, donde la principal tarea es comprender, memorizar y el nivel de aplicación es bajo, transformándose a un contexto donde además de comprender, tienen que buscar una aplicación del conocimiento obtenido en los aspectos clínicos, aunado a que inician el contacto con los pacientes y necesitan además adquirir habilidades en su capacidad de comunicación, abstracción y análisis ${ }^{16-18}$.

Otros investigadores han encontrado que los últimos años de la carrera generan también una elevada proporción de estrés, en los estudiantes, por que los mismos se enfrentan en el siguiente año, al internado médico, donde el nivel de responsabilidad sobre la atención de los pacientes es más directo ${ }^{19}$.

En este trabajo nosotros quisimos conocer si el nivel de ansiedad y depresión es mayor en los alumnos de medicina que se encuentran cursando su internado médico comparado con los estudiantes del último año de la carrera de medicina, encontrando que la prevalencia de ansiedad es mayor en el internado que en el último año de la carrera, aunque el nivel de ansiedad es significativamente mayor en estos alumnos que el reportado para la población general en México, lo cual nos permite concluir que el último año de la carrera también es un factor generador de elevada ansiedad y que es significativamente mayor que el de la población general, sin embargo no podemos concluir si es mayor que en otros momentos de la carrera.

Así mismo, nosotros encontramos una prevalencia de ansiedad similar a la reportada en otros estudios, realizados tanto en países desarrollados como en vías de desarrollo, lo cual nos hace pensar que éste es un problema inherente a la formación de los médicos y que aunque influyen ciertos factores propios de cada país y sistema de salud 2,10,13 los desencadenantes de los mismos son como los considera Morrison y colaboradores, la elevada carga académica, la presión social y los problemas económicos ${ }^{20}$.

Otro dato relevante es el hecho de que encontramos una elevada proporción de depresión en los estudiantes del internado y que el nivel de depresión de los estudiantes del último año de la licenciatura, es similar al de la población general. Esto traduce que los factores que inciden en que alumnos que previamente presentaban un elevado nivel de ansiedad, encuentran en el primer año de su contacto directo con los pacientes, suficientes factores desestabilizadores, para progresar hacia un estado de depresión, los cuales pueden ser explicados porque los internos requieren desarrollar habilidades para adaptarse a un nuevo sistema organizacional, un nuevo nivel de responsabilidad en el cuidado del paciente, y de comunicación con los pacientes, aunado a que se mantienen actividades de aprendizaje y enseñanza.

Si bien la frecuencia de depresión es significativamente mas elevada en los internos de pregrado en comparación con la de los estudiantes del último año de la carrera es muy similar a la reportada por otros autores en residentes de primer año $0^{17,19,21,22}$.

Esto tiene muchos aspectos a considerar y que deben ser estudiados de una manera prospectiva, para identificarlos primero y después incidir en ellos; sin embargo es in- 
dudable que este elevado nivel de ansiedad y depresión representan un riesgo para el paciente, ya que los médicos que manejan inadecuadamente el estrés, pueden tomar decisiones vitales incorrectas para el paciente. Aunque debemos reconocer que el nivel de responsabilidad del médico interno en el sistema de salud mexicano es bajo, es indudable que el médico interno representa un elemento relevante en la atención global del paciente hospitalizado, de tal manera que quizá, el diseño de estrategias para que los médicos internos o los estudiantes de medicina aprendan a realizar un

\section{Bibliografía}

1 Weissman $M M$, Recent advances in depresión across the generations. Epidemiol Psichiatr Soc; 2006; 15:16-19

2 Castilla-Puentes RC, secin R, Grau A, Galeno R, Freijo de Mello M, Pena N, Sanchez-Russi CA. A multicenter study of major depressive disorder among emergency departamento patients in Latin-American countries. Depress Anxiety. 2008;25(12):E199-204.

3 http://www.who.int/mental_health/management/ depression/definition/en/

4 Pascual Báez P, de la Cruz J, Feliz Alcántara M. Carácterización de los trastornos depresivos en una población de estudiantes de medicina. Revista Latinoamericana de Psiquiatría. 2007; sept p50

5 Joffre-Velázquez VM, Martínez-Perales G, GarcíaMaldonado G, Sánchez-Gutiérrez de Lara L. Depresión en estudiantes de medicina. Resultados de la aplicación del invetario de depresión de Beck en su versión de 13 ítems. Alcmeon, Revista Argentina de Clínica Neuropsiquiátrica, 2007; 16(14):86-93

6 Lugo M., Lara C, González JE, Granadillo D., Depresión, ansiedad y estrés en estudiantes de medicina del área básica y clínica, su relación con el índice de lateralización hemisférica cerebral y el rendimiento académico. Universidad de Carabobo año 1999-2000. Archivos venezolanos de Psiquiatría y Neurología. 2004;50(103)21-29

7 Dunn LA, Iglewicz A, Moutier C. A conceptual model of medical student well-being: Promoting resilence and preventing Burnout. Acad Psychiatry. 2008;32:44-53.

8 Dyrbye LN, Thomas MR, Huntington JL, et al: Personal life events and medical student burnout: a multicenter study. Acad Med 2006; 81:374-384.

9 Kiessling C, Schubert B, Scheffner D, et al: First year medical students' perceptions of stress and support: a comparison between reformed and traditional track curricula. Med Educ 2004; 38:504-509

10 Omigbodun OO, Odukogbe AT, Omigbodun AO, Yusuf OB, Bella TT, Olayemi O. Stressors and psychological symptoms in students of medicine and allied health professions in Nigeria. Soc Psychiatry Psychiatr correcto manejo del estrés pueda mejorar su rendimiento y esto pueda incidir en una mejor atención hacia sus pacientes ${ }^{23}$.

Una importante limitación del presente estudio es que con un diseño transversal, no podemos hacer inferencias sobre los factores que determinan una mayor prevalencia de depresión en los médicos internos; pero sí podemos considerar con suficiente fundamento, que los factores que generan este incremento están presentes en los hospitales, por lo cual debemos diseñar trabajos de investigación que determinen cuales son esos factores en nuestro medio.

Epidemiol. 2006;41(5):415-421.

11 Stewart SM, Betson C, Lam TH, et al: Predicting stress in first year medical students: a longitudinal study. Med Educ 1997; 31:163-168

12 Ball S, Bax A: Self-care in medical education: effectiveness of health-habits interventions for first-year medical students. Acad Med 2002; 77:911-917.

13 Drybye LN, Thomas MR, Shanafelt TD. Systematic review of depression, anxiety, and other indicators of psychological distress among U.S. and Canadian medical students. Acad Med. 2006:81(4):354-373.

14 Hafen M Jr, Reisbig AM, White MB, Rush BR. The firstyear veterinary student and mental health: the role of common stressors. Vet Med Educ 2008;35(1):102109.

15 Guthie EA, Black D, Shaw CM, Hamilton J, Creed FH, Tomenson B. Embarking upon a medical career: Psychological morbidity in first year medical student. Med Educ. 1995;29:337-341.

16 Radclife $\mathrm{C}$, Lester $\mathrm{H}$. Perceived stress during undergraduate medical training: a qualitative study. Med Educ. 2003:37:32-38.

17 Sung $A D$, Collins $M E$, Smith AK, Sanders AM, Quinn $M A$, Block SD, Arnold RM. Crying: experiences and attitudes of third-year medical students and interns. Teach Leran Med. 2009;21(3):180-187.

18 Ahmed I, Banu H, Al-Fageer R, Al-Suwadi R. Cognitive emotions: depression and anxiety in medical students and staff. J Crit Care. 2009;24(3):e1-7.

19 Schneider SE, Philips WM. Depression and anxiety in medical, surgical, and pediatric interns. Psychol Rep. 1993;72(3 Pt2):1145-1146.

20 Morrison J. More on medical students stress. Acad Med. 2001;35:617-618.

21 Peterlini M, Tiberio IF, Saadeh A, Pereira JC, Martins MA. Anxiety and depression in the first year of medical residency training. Med Educ. 2002;36(1):66-72.

22 Buddeberg-Fischer B, Klaghofer R, Buddeberg C. Stress at work and well-being in junior residents. $Z$ Pyscchosom Med Psychother. 2005;51(2):163-178.

23 LeBlanc VR. The effects of acute stress on performance: implications for health professions education. Acad Med. 2009;84(10 Suppl):S25-S33. 